\title{
La professionnalisation de la prostitution : Le travail des femmes (aussi) en question
}

Pascale ABSI

Les débats autour de la professionnalisation de la prostitution ont stimulé son approche en termes de travail, de métier de service, de réelle alternative économique, d'utilité sociale, requérant des compétences particulières. D'une part, de plus en plus de personnes prostituées revendiquent, à travers le monde, le statut de travailleur du sexe pour appuyer leur demande de reconnaissance et de droits sociaux, comme c'est le cas du nouveau syndicat français du travail du sexe (Strass). D'autre part, l'analyse de la prostitution s'est en partie extraite du champ de la déviance et de la violence faite aux femmes pour mobiliser les outils de la sociologie du travail et des professions, sans nécessairement d'ailleurs prendre position en faveur de la professionnalisation ${ }^{1}$. Dans les deux cas, qu'elle s'adresse au juridique ou à la sociologie, le rattachement de cette activité au monde du travail défit la conception fondamentalement antiprofessionnalisante de la prostitution comme asservissement sexuel, symbole par excellence de l'oppression patriarcale qui s'exerce sur les femmes.

La controverse ne mobilise pas uniquement des perceptions contradictoires de l'expérience et de l'exercice de la prostitution. On le néglige souvent, des acceptations diverses de ce qu'est le travail entrent également en jeu. À quelle(s) conception(s) différentielles du travail, notamment celui des femmes, renvoient l'inclusion ou l'exclusion de la prostitution? La question prolonge à sa manière l'examen magistral par Paola Tabet des multiples déclinaisons des échanges économico-

1. Cf. par exemple : Wendy CHAPKIS, Live Sex Acts : Women Performing Erotic Labor, Routledge, New York, 1997 ; Stéphanie PRYEN, Stigmate et métier. Une approche sociologique de la prostitution de rue, Presses Universitaires de Rennes, 1999 ; Colette PARENT et Chris BRUCKERT, «Le travail du sexe dans les établissements de services érotiques : une forme de travail marginalisée », Déviance et société, vol. 29, n 1, p. 33-53.

L'homme et la société, $\mathrm{n}^{\mathrm{o}}$ 176-177, avril-septembre 2010 
sexuels ${ }^{2}$. Chercher à y répondre ne révolutionnera pas le débat sur le statut de la prostitution. En le décentrant, l'entrée par les imaginaires du travail peut cependant contribuer à éviter l'écueil du « pour ou contre la prostitution" dans lequel il se perd parfois. Surtout, elle permet d'enrichir la compréhension des visions du monde qui s'y affrontent. Sans oublier celles des chercheurs qui, du choix des groupes sociaux et des activités prises en compte aux catégories d'analyses et au rôle présumé du travail dans le "faire société », véhiculent des conceptions implicites lorsqu'ils identifient certaines tâches comme travail tout en en excluant d'autres, indépendamment de la parole des acteurs. Utiliser les discours sur le statut de la prostitution comme analyseurs des représentations du travail conforte ainsi le rôle heuristique de cette pratique pour la compréhension des normes sociales, comme c'est déjà le cas pour les rapports de sexes ou, plus récemment, la marchandisation ${ }^{3}$.

Mon interrogation sur la polysémie des représentations du travail mobilisées par le débat sur la professionnalisation a pris corps lors d'un terrain ethnographique dans les maisons closes populaires de Bolivie. C'était en 2004, je débutais alors une recherche sur les représentations du travail et de l'argent des travailleurs précaires de la ville de Potosi. Quelques mois plus tard, j'assistais à la naissance de la première organisation nationale des prostituées boliviennes ${ }^{4}$ sous l'égide de la principale confédération syndicale du pays. L'événement prolongeait l'initiative des organisations de santé publique et des associations de droits de l'homme d'organiser les femmes des maisons closes. Il marqua l'entrée des prostituées boliviennes dans le débat. L'occasion aussi d'interroger les définitions contradictoires du travail des différents acteurs de ce processus selon la place accordée au revenu, à la nature de l'activité et aux rapports sociaux : les personnels de santé financés par l'ONUsida et l'Organisation panaméricaine de la santé (OPS), un collectif féministe, des associations de défense des droits, les syndicalistes et, bien sûr, les prostituées elles-mêmes.

2. Paola TABET, «Du don au tarif. Les relations sexuelles impliquant une compensation », Les Temps modernes, 1987, vol. 42, n 490, p. 1-53 et La grande arnaque. Sexualité des femmes et échange économico-sexuel, L'Harmattan, «Bibliothèque du féminisme », 2004.

3. Philippe Alonzo, Tania Angeloff et Delphine GARdey (éds.), Travail, genre et société, $\mathrm{n}^{\circ} 10,2003 / 2$, "Prostitution : marchés, organisation, mobilisations »; Martha ERTMANN et Joan Williams, Rethinking Commodification : Cases and Readings In Law and Culture, New York University Press, 2005.

4. La prostitution masculine est peu répandue en Bolivie, les hommes et les transsexuels sont exclus des organisations étudiées, ce qui explique que cet article se réfère le plus souvent aux « prostituées ». 
L'exercice, dont les résultats sont exposés ici, fait sien le rappel que la prostitution est «une pratique avant de pouvoir être érigée en théorie ${ }^{5} »$. Une telle perspective implique de «prendre au sérieux la parole des prostitués ${ }^{6}$ et de laisser de côté les préconcepts universalisant pour réinterroger sa complexité en fonction des trajectoires individuelles, des conditions d'exercice mais aussi des contextes locaux où s'ancrent les imaginaires de la prostitution, et donc du travail.

\section{L'entrée et la vie dans le milieu?}

La Bolivie est un pays réglementariste en matière de prostitution. Dans les maisons closes, son exercice est sujet à un examen médical obligatoire et au port d'un carnet de santé contrôlé lors de battues.

La plupart des prostitués sont des femmes, jeunes. Elles ont généralement entre 20 et 30 ans, ce qui tend à prouver qu'il y a une autre vie après la prostitution. Elles proviennent surtout des zones urbaines de l'ensemble du pays mais les femmes des basses terres sont plus présentes que celles de la cordillère et des hauts plateaux. Leurs parents possèdent un petit commerce de rue, parfois un atelier, et sont plus rarement agriculteurs ou mineurs. Si les femmes entrent jeunes en prostitution, souvent entre 17 et 20 ans, celle-ci succède presque toujours à un parcours professionnel qui a commencé bien plus tôt, vers 12-13 ans, dans l'emploi domestique, lequel ne signifie pas toujours l'arrêt des études. La succession d'emplois mal rémunérés, des relations familiales difficiles, la sortie de la domesticité et du contrôle des patrons, l'attrait de la liberté et des distractions juvéniles puis une grossesse précoce, sont des enchaînements récurrents sur le parcours qui débouche sur la prostitution. L'impossibilité de rembourser une dette ou un micro-crédit est également un facteur important. La rencontre avec une "amie» qui éblouit les jeunes femmes avec son argent avant de leur proposer de travailler comme serveuse dans une maison close - il s'agit parfois d'une intermédiaire qui touche une commission - est presque toujours présentée comme l'événement déclencheur de l'entrée en prostitution ${ }^{8}$.

5. Catherine Deschamps et Anne Souyris, Femmes publiques. Les féminismes à l'épreuve de la prostitution, Paris, éd. Amsterdam, 2008, p. 17.

6. Stéphanie PRYEN, Stigmate et métier. Une approche sociologique de la prostitution de rue, op. cit., p. 24.

7. Le terme espagnol « el ambiente » est utilisé pour désigner « le milieu » de la prostitution. Les informations sur l'origine sociale et géographique des prostituées sont issues d'une enquête plus vaste en cours de publication, menée avec le géographe Hubert Mazurek.

8. Parfois, les propriétaires recrutent eux-mêmes dans les discothèques ou les gares routières, voire par le biais d'agences de placement sous couvert d'emplois de serveuse. 
Une fois sur place, l'emploi de serveuse devient un emploi d'entraineuse puis de prostituée.

En échange du respect des horaires (5 à 7 heures par nuit), les femmes peuvent bénéficier d'une chambre. Elles y reçoivent certains clients bien qu'il existe des pièces réservées aux relations sexuelles. D'autres femmes ont un domicile en ville où elles vivent avec leurs enfants et parfois leur conjoint. Elles peuvent alors choisir leurs horaires et leurs jours de travail. Dans la ville minière de Potosi, où il n'existe que des établissements populaires, le prix de la relation sexuelle est fixé par l'usage à 5 euros. Ce tarif de base peut être négocié (à la hausse comme à la baisse) avec les femmes qui reversent 1 euro au tenancier. Les prostituées touchent également une commission sur la consommation d'alcool de leurs clients. On est donc dans un système de travail à la pièce où, du moins, sur le terrain de l'enquête, les femmes subissent rarement de pressions quant au nombre de leurs clients. Bien que supérieurs à ceux de l'emploi domestique (environ 50 euros par mois), les revenus de la prostitution restent précaires. Pour y remédier, les prostituées suivent les flux économiques : l'ouverture d'une entreprise minière ou la réactivation de la production de coca par exemple, motivent la migration d'un lieu à l'autre. Depuis la fin des années 1980, les femmes sont libres de quitter les maisons closes quand bon leur semble. L'ouverture à la concurrence incite désormais les tenanciers à mieux traiter leurs employées par peur de voir leur établissement déserté. Une part importante des revenus est destinée aux enfants souvent restés chez leurs grands-parents. D'autres femmes économisent pour se faire construire une maison ou ouvrir un commerce. Mais mener une vie instable coûte cher : une grande partie de l'argent finit en dépenses quotidiennes et festives. Ce n'est qu'avec l'âge que les femmes commencent à préparer l'avenir, cependant, leurs gains sont alors bien moins importants. La sortie de la prostitution passe souvent par la mise en ménage avec un homme qui apporte parfois le capital nécessaire à l'ouverture d'un commerce ou la reprise d'une maison close. Au risque de s'y asservir, le désir des femmes de mener une vie conventionnelle rencontre alors la fantaisie masculine de les « sortir de là »...

Les histoires de vie des femmes que j'ai rencontrées sont tissées de violences morales, physiques et sexuelles, de précarités affectives et économiques. Sur ce plan pourtant, le quotidien des maisons closes ne présente pas une grande nouveauté. L'entrée en prostitution peut même favoriser une autonomie financière qu'interdisaient aux femmes leurs salaires d'enfant et leur dépendance des hommes, père ou mari, dont le rôle de pourvoyeur de revenus assure la domination. La prostitution permet 
alors de renégocier leur place dans la société mais aussi au sein de leur famille qu'elles aident économiquement. Ces remarques n'affirment pas que la prostitution soit la voie de la libération. Comme le suggère Paola Tabet, comparer « les degrés de contraintes ou d'autonomie des femmes dans les diverses formes de relations a un sens précis : c'est respecter, essayer de comprendre et d'analyser les choix que font les femmes ellesmêmes, même si ces choix demeurent tous à l'intérieur des systèmes de domination masculine et ne permettent pas d'y échapper ${ }^{9} »$. Une démarche qui souligne l'importance de la mise en relation, sur un terrain donné, de l'expérience de la prostitution avec la vie probable des femmes si elles étaient restées en dehors. Replacer la prostitution dans les trajectoires de vie suit également l'enseignement des sociologues du travail qui soulignent que le vécu des personnes ne peut jamais s'appréhender au travers d'une seule sphère de leur vie, fut-elle aussi prégnante que la prostitution. Il existe ainsi une sorte de "violence interprétative $^{10}$ » à analyser l'expérience des prostitués uniquement au travers du commerce sexuel.

\section{L'organisation des prostituées et le rapprochement avec la Centrale ouvrière}

Le projet d'une «organisation corporative» des prostituées boliviennes surgit lors des « Deuxièmes rencontres latino-américaines de femmes travailleuses sexuelles » organisées en 1998 à Santa Cruz, grâce à l'appui de USAID qui soutient les centres de dépistage des maladies sexuellement transmissibles où les prostituées effectuent leurs contrôles médicaux. Les mauvais traitements des tenanciers, des policiers, du personnel médical et l'obligation de fichage motivèrent l'envoi de lettres de doléances au Défenseur du peuple ${ }^{11}$. Trois ans plus tard, cette institution organise, à Cochabamba, la «Première rencontre nationale de travailleuses sexuelles» en présence du secrétaire de la COD (Centrale ouvrière départementale) ${ }^{12}$. Quelques temps plus tard, en janvier 2004, le

9. Paola TABET, La grande arnaque. Sexualité des femmes et échange économicosexuel, op. cit., p. 118.

10. J'emprunte l'expression à Claudia Girola (« Du don à la transaction : une économie morale. Le cas des personnes sans abri », Communication au colloque "Économie informelle, travail au noir», Université de Marne-La-Vallée, 17 septembre 2007) qui l'utilise pour se référer à la manière dont certaines études postulent que le vécu des SDF est tout entier orienté par la satisfaction de leurs besoins primaires.

11. La «Defensoría del Pueblo» est une institution publique de médiation entre les citoyens et l'administration.

12. La Centrale ouvrière bolivienne $(\mathrm{COB})$ fédère les différentes Centrales ouvrières départementales (COD). 
secrétaire permanent de la COD d'Oruro offre son appui aux prostituées. S'ensuit l'élection d'un directoire des Travailleuses sexuelles d'Oruro dont la présidente, Luz, va organiser ses paires dans tout le pays. Inspiré par le précédent argentin ${ }^{13}$, l'objectif est la constitution d'une organisation nationale de travailleuses sexuelles en vue de son affiliation à la Centrale ouvrière nationale (COB). En décembre 2005, la COD d'Oruro inaugure, avec un financement de l'Organisation panaméricaine de la santé (OPS), le « $\mathrm{I}^{\text {er }}$ Congrès national de l'organisation des travailleuses sexuelles commerciales $»$.

L'organisation permit la suppression du fichage policier (2001) et la gratuité des examens médicaux obligatoires (2004). Le rapprochement avec le plus grand syndicat du pays conforta également la recherche de légitimité des femmes, et certaines dirigeantes commencèrent à apparaître à visage découvert. Entre rires et blagues, " exiger nos droits » se convertit en refrain et les femmes s'approprièrent les classiques instruments des luttes sociales boliviennes: les manifestations, les barrages routiers et les grèves de la faim. Mais le processus d'affiliation s'interrompit lorsqu'une partie du syndicat refusa l'entrée des prostituées pour contrarier les intérêts des dirigeants qui les appuyaient. Elles n'intégrèrent jamais la $\mathrm{COB}$ mais leur chemin venait de rencontrer le débat international sur la professionnalisation.

\section{Travail ou activité ? Le discours des institutions}

L'apparition successive de différents acteurs institutionnels dans l'organisation des prostituées est corrélative au changement de lieu d'où celles-ci formulèrent leurs revendications: en tant que travailleuses sexuelles dans le cadre sanitaire des programmes ITS/Sida, puis comme citoyennes qui exercent la prostitution, avant de faire irruption dans le droit du travail puis d'être remisées au rang de pratiquantes d'une activité. Ces allées et venues témoignent de la difficulté pour les prostituées boliviennes de construire une position propre alors qu'elles sont sans cesse soumises à des exhortations contradictoires de la part des différentes institutions qui interviennent dans leur secteur.

J'en résumerai ici les principales positions recueillies lors d'entretiens avec leurs responsables et de l'observation des prises de position auprès

13. AMMAR, la principale association de prostituées en Argentine, est affiliée à la Centrale des travailleurs argentins depuis 1995. Sa dirigeante historique, Elena Reynaga, est venue plusieurs fois en Bolivie rencontrer ses paires. Aujourd'hui, une partie d'AMMAR s'est séparée de l'organisation mère par rejet de la syndicalisation et de la professionnalisation, et ses dirigeantes se sont rapprochées du collectif féministe bolivien Mujeres Creando. 
des prostituées. Le caractère réducteur de ce résumé, qui omet les subtilités et les dissensions internes, ne devrait pas invalider la démonstration de la diversité des conceptions du travail mobilisées par le débat sur la professionnalisation. Des ponts apparaissent avec les termes du débat ailleurs dans le monde, notamment en France ${ }^{14}$, même si le réglementarisme bolivien et l'existence de maisons closes favorisent certainement la conceptualisation de la prostitution comme rapport de travail.

\section{La position économiciste de l'Organisation internationale du travail}

Dans son rapport controversé de $1998^{15}$, l'Organisation internationale du travail (OIT) appelle à la reconnaissance de l'industrie du sexe, soulignant l'expansion de la prostitution et de sa contribution non reconnue à l'emploi et au PNB. Cette reconnaissance englobe une extension des droits du travail pour les travailleurs sexuels et une incitation pour les gouvernements à tirer avantage des énormes bénéfices de l'industrie du sexe moyennant prélèvement fiscal. En Bolivie, la posture de l'OIT est relayée par les autres instances de l'ONU liées au secteur de la prostitution. C'est le cas de l'OPS et de l'ONUSida qui appuient le programme bolivien MST/Sida, responsable du suivi médical des prostituées. Ce n'est donc pas un hasard si son personnel a largement participé à la diffusion de l'appellation « travailleuses sexuelles » depuis les années 1990. En 2005, le programme national imprima une série d'autocollants proclamant «N'aies pas honte d'être une prostituée, femme, tu as une profession » et « Donne de la valeur à ton travail ${ }^{16}$.

La posture de l'OIT postule le caractère inévitable de la prostitution et souligne qu'elle constitue une option plus lucrative et flexible que d'autres emplois féminins. De tels arguments déclenchèrent évidemment la colère d'associations féministes qui fustigèrent l'opportunisme et le cynisme

de l'OIT défendant l'idée de la prostitution comme un choix de travail rationnel et la transformation de l'exploitation sexuelle et économique des

14. Lilian Mathieu, Mobilisations de prostituées, Paris, Belin, coll. « Socio-histoires », 2001.

15. The Sex Sector: the Economic and Social Base of Prostitution in Southern Asia, Lin Lean LiM (dir.), BIT, Genève, 1998. Voir aussi l'interview de Lin Lean Lim, bulletin Travail, 26, OIT, sept/oct 1998.

16. Cette initiative avait d'ailleurs valu son poste à la directrice du programme suite à la plainte de l'association des prostituées de La Paz qui s'étaient laissées entraîner par les tenants de l'anti-professionnalisation (Centre de nouvelles OPS/OMS Bolivia, 15/11/05). 
femmes en travail légitime ${ }^{17}$. En Bolivie, des critiques similaires alimentent la posture d'ONG comme Sayari Warmi qui collabora activement à l'organisation des prostituées et du collectif féministe Mujeres Creando qui intégra plusieurs dirigeantes du secteur de la prostitution.

\section{Sayari Warmi : le droit du travail pour un non travail}

Sayari Warmi (du quechua, «femme, lève-toi ») est une œuvre catholique qui mène un programme de lutte contre la violence faite aux femmes et leur propose des formations professionnelles. Dans ce cadre, l'association a soutenu l'organisation des prostituées et contribué à la réalisation de leur premier congrès national.

Pour les responsables de Sayari Warmi, il ne fait aucun doute que la prostitution ne peut être définie comme un travail. La première raison invoquée est l'absence de contrat de travail et de bénéfices sociaux correspondants (sécurité sociale, retraite...). Paradoxalement, l'autre renvoie à la conception par les intégrants de Sayari Warmi de la prostitution comme un esclavage sexuel imbriqué avec le patriarcat et l'économie de marché, ce qui exclut a priori son institutionnalisation. Leur posture conjugue ainsi de manière ambiguë — comment solliciter le droit du travail si ce que l'on fait ne peut pas être reconnu comme travail ? - le projet du néo-réglementarisme (la prostituée comme travailleuse) et de l'abolitionnisme. Elle est partagée par d'autres institutions comme l'Assemblée des droits humains et la Defensoria del Pueblo qui interviennent lors des conflits qui opposent les prostituées aux autorités sanitaires ou policières.

\section{Le féminisme de Mujeres Creando}

Mujeres Creando (Femmes qui créent) est un collectif féministe qui mène depuis de nombreuses années une réflexion sur la prostitution, avec des prostituées parmi lesquelles les dissidentes argentines qui refusèrent la syndicalisation de leur association ${ }^{18}$. S'il partage la même analyse féministe de la prostitution que celle de Sayari Warmi, ses membres ont toujours veillé à conceptualiser les revendications des prostituées en termes de droits citoyens et non de droits du travail.

Ainsi, le rapport de la rencontre «Aucune femme ne naît pour être pute » de 2003, établit clairement que les signataires — parmi lesquels des prostituées - considèrent que la prostitution convertit les femmes en

17. Janica RAYMOND, «Légitimer la prostitution en tant que travail. L'OIT appelle à la reconnaissance de l'industrie du sexe », 1998 : sisyphe.org/article.php3?id article=158

18. Cf. note 13 et Mujeres Creando, La Virgen de los Deseos, Ediciones Tinta Limón, Buenos Aires, 2005. 
« marchandises » plus qu'en « travailleuses ${ }^{19}$. La prostitution n'est pas une activité librement choisie mais le résultat de la violence exercée sur les femmes notamment celle du chômage qui, dans le contexte patriarcal de la société bolivienne, les affecte tout particulièrement. La reconnaissance de la prostitution comme travail libère l'État de son obligation de créer de véritables emplois pour les femmes. Le document mentionne également la violence de l'expérience prostitutionnelle, le vieillissement prématuré des femmes et leur stigmatisation.

\section{Les doutes conceptuels de la Centrale ouvrière}

La position des syndicalistes sur le rapprochement avec les prostituées est exemplaire $\mathrm{du}$ pouvoir des discours sur la professionnalisation de révéler des représentations du travail. La Centrale ouvrière bolivienne (COB) traversait l'une des principales crises de son histoire lorsqu'elle décida d'affilier les prostituées. La décomposition du monde syndical suite à l'ajustement structurel des années $1980^{20}$ et à la fermeture des mines d'État ont profondément déstabilisé le secteur minier qui constitue historiquement la principale force vive de la Centrale ouvrière ${ }^{21}$. Les privatisations et les licenciements qui multiplièrent les travailleurs à leur compte et le chômage contribuèrent également à altérer la base classiste traditionnelle de la confédération syndicale. Le déplacement des ouvriers par les paysans et les indigènes qui portèrent Evo Morales ne fit qu'intensifier la crise d'identité de la Centrale ouvrière. Le rapprochement avec les prostituées coïncidait ainsi avec la nécessité de la $\mathrm{COB}$ de flexibiliser ses anciens modèles d'affiliation, comme celui d'être sujet à une relation patronale, et d'élargir sa juridiction vers les Droits de l'homme. Cette stratégie fut officialisée en juillet 2004 dans la thèse politique de son XIII ${ }^{\mathrm{e}}$ Congrès national, «Récupération et renforcement de la $\mathrm{COB}$ ». Cinq mois plus tard, David Rojas, alors secrétaire permanent de la COD d'Oruro, commentait le processus de la sorte :

«La classe ouvrière a diminué et si la classe ouvrière n'est plus présente, nos mobilisations doivent évidemment se recentrer sur les secteurs de la classe moyenne, des enseignants, des professionnels de la santé et des paysans. [...]

19. MujerES CREANDO, ibidem.

20. Álvaro GARCIA LinERA (coord.), Sociología de los movimientos sociales en Bolivia. Estructuras de movilización, repertorios culturales y acción política, Diakonia/Oxfam G.B/Plural, La Paz, 2004, p. 71-72.

21. La Centrale ouvrière bolivienne, de tendance marxiste, est une confédération de syndicats née de la révolution de 1952, menée par les mineurs contre l'oligarchie. Elle s'est constituée comme l'un des syndicats les plus puissants d'Amérique latine. 
Nous devons unir la lutte des travailleurs avec les secteurs du peuple contre le modèle [néolibéral]. Nous sommes en train de parler de tous les secteurs déprimés par le modèle, à partir de là, on accepte l'entrée des travailleuses sexuelles. »

L'affiliation des «travailleuses sexuelles » s'inscrivait ainsi dans le processus d'ouverture qui permit l'entrée de non-ouvriers (notamment les petits commerçants autrefois considérés comme des petits bourgeois), de secteurs peu reconnus dans le monde du travail comme les employées de maison et, plus récemment, de collectifs non professionnels de sans-abri ou de petits épargnants ruinés par l'escroquerie bancaire ${ }^{22}$. Tous partagent le statut de victime du système néolibéral comme ticket d'entrée à la COB. Particulièrement emblématiques des désajustements du monde du travail, les prostituées se sont ainsi transformées en porte-drapeaux de l'oppression libérale. La rhétorique dissout le genre: jamais les dirigeants masculins n'évoquèrent la place particulière des femmes sur le marché du travail.

Plus pragmatiquement, l'entrée des prostituées répondait à la stratégie du principal dirigeant de gagner des votes en vue de sa réélection. Selon les mécanismes électoraux de la Centrale ouvrière, une fois affiliées, le vote des prostituées aurait eu le même poids que celui de n'importe quel autre secteur. Mais encore fallait-il décider en tant que quoi ces femmes étaient victimes du système économique : l'étaient-elles comme travailleuses ou comme simples citoyennes? Au final, la Centrale ouvrière refusa aux prostituées le statut de travailleuses comme l'expliquait David Rojas dans la suite de notre entretien de 2004 :

«Nous ne considérons pas que cela soit un travail dans le sens strict du terme. Nous considérons qu'il s'agit des niveaux de dégradation les plus extrêmes impulsés par ce modèle, le système [néolibéral]. [...] Ce n'est pas un travail parce qu'il ne génère pas de plus-value, il ne génère pas de capital, il ne mobilise pas de capital ; la tirelire est en elles-mêmes n'est-ce pas ? Elles ne vendent pas non plus de force de travail, c'est une activité. Il y a des débats, certains camarades disent que si, c'est un travail, le dernier recours des camarades femmes. Mais moi, personnellement, je refuse de l'accepter comme travail. Je pense que notre travail, comme dirigeants syndicaux, devrait se centrer, devrait être celui de promouvoir un processus de sortie de ces camarades de la prostitution. »

De manière assez caricaturale, ce témoignage illustre toute la difficulté des dirigeants syndicaux de renoncer à leurs outils conceptuels traditionnels pour repenser ce que sont actuellement un travailleur et un travail. Malgré la volonté manifeste de s'ouvrir aux «nouveaux prolétaires », les définitions en termes de « plus-value » ou de « capital»

22. De fait, les prostituées devaient être affiliées comme représentantes de la classe moyenne, aux côtés des commerçants et des mineurs coopérateurs, par distinction avec le prolétariat ouvrier. 
en excluent de fait le plus grand nombre. Que penser aussi de l'argument selon lequel les prostituées ne « vendent [pas] leur force de travail »- à l'image d'un commerçant — qui reflète une vision de la prostitution comme une pratique passive, en adéquation avec la représentation traditionnelle du rôle sexuel de la femme et l'association de l'effort physique avec la masculinité. L'absence de patron, le travail à son compte, l'idée que les prostituées sont «maîtresses de leurs moyens de production »: des arguments semblables sont utilisés par la COB pour maintenir les secteurs non ouvriers en marge du pouvoir syndical, y compris lorsque ceux-ci rassemblent plus d'adhérents que le secteur minier qui fournit, à la confédération syndicale ses principaux dirigeants.

Le refus de la reconnaissance de la prostitution comme travail fut exporté par la COD Oruro vers la commission politique du « $\mathrm{I}^{\mathrm{er}}$ Congrès national des travailleuses sexuelles» qu'elle organisa et motiva l'abandon de l'appellation «travailleuses sexuelles». La nouvelle association nationale qui surgit du congrès fut baptisée «Association nationale d'activistes pour l'émancipation de la femme». Le terme d'activistes répondait à la volonté de penser la prostitution comme une activité et non comme un travail. Son ambiguïté rendit invisible la relation entre l'association et la prostitution. Tel était le prix à payer pour pouvoir figurer de manière « décente » sur les listes de la COB.

Ce bref tour d'horizon confirme l'usage polysémique et la nature idéologique du concept de travail. L'OIT défend une approche économiciste où le revenu monétaire définit le travail. Cette vision s'oppose à celle des féministes qui dénoncent les rapports sociaux à l'origine des revenus de la prostitution; sa conceptualisation comme esclavage sexuel conteste alors toute possibilité de professionnalisation. $\mathrm{La}$ rhétorique des dirigeants de la Centrale ouvrière conditionne également l'existence du travail à la présence d'un certain type de rapports sociaux; mais c'est alors l'absence (supposée) de domination patronale qui contrarie sa reconnaissance comme travail. Sur ce point, les prostituées ne sont pas d'accord avec les syndicalistes. En même temps, elles sont socialement plus proches d'eux et partagent des référents communs, notamment, nous le verrons, l'effort physique productif. D'ailleurs, à la différence du Syndicat français des travailleurs du sexe (STRASS), les organisations de prostituées boliviennes n'ont jamais pensé à se proclamer syndicat.

\section{Mais que pensent les femmes concernées?}


Pour la plupart des femmes qui l'exercent en Bolivie, il ne fait aucun doute que la prostitution est un travail puisque c'est une source de revenus. "Si ce n'était pas un travail, nous le ferions gratuitement " plaisante, à moitié, Evelyn. Elles adoptèrent avec enthousiasme le nom de « travailleuses sexuelles », et la possibilité de s'affilier à la Centrale ouvrière officialisa leur sentiment intime. Mais celles des femmes qui participent aux réunions et ateliers des institutions qui travaillent avec les prostituées, doivent désormais composer avec le discours contre la professionnalisation.

\section{La réception des arguments du non-travail}

La conception de la prostitution comme symbole de la domination masculine et de son indignité limite l'intériorisation par les femmes du discours contre la professionnalisation. "D'autres disent que nous ne sommes pas des travailleuses, que sommes-nous alors? Des objets? Je ne me sens pas non plus être un objet », critique Mechi, une dirigeante de longue date. Assumer un statut de victime des hommes et du système peut paraître encore plus humiliant que celui de « pute » et ne correspond pas à la praxis des femmes expérimentées ${ }^{23}$. Le pouvoir relatif de ces femmes sur le choix de leurs tarifs, de leurs clients et de leurs services, leur accorde une sensation de contrôle voire de domination. Rabaisser l'ego du client en l'humiliant et lui extorquer de l'argent sont des pratiques courantes; de même que le vol, perçu comme l'annulation ludique du pouvoir de l'argent des hommes. À aucun moment ces pratiques ne s'inscrivent dans un discours plus large sur la domination masculine. Elles visent à établir au cas par cas une relation équilibrée avec des hommes envers lesquels les prostituées démontrent finalement une grande loyauté. La dépendance des prostituées boliviennes du modèle de la femme au foyer et la possibilité qu'elles s'autorisent de tisser des liens affectifs avec leurs clients réguliers, voire de les épouser, les incitent à refuser avec force les discours qui construisent le client comme une catégorie par nature hostile là où elles ne voient que des individus à classer en bons et mauvais clients. De fait, c'est principalement l'absence de droits associés au travail qui a convaincu les dirigeantes que leur activité ne pouvait être considérée comme telle. Le premier objectif de l'affiliation à la COB était ainsi d'obtenir ces droits par lesquels viendrait leur reconnaissance comme travailleuses. Mais la Centrale ouvrière convainquit les femmes de renoncer à ce projet. Lors

23. La situation des mineures qui exercent dans la clandestinité est beaucoup plus vulnérable. 
du congrès organisé sous son égide, la perte d'autonomie que signifierait la transformation de la prostitution en relation de travail contractuelle devint l'argument décisif de Luz, candidate à la présidence nationale, pour convaincre ses bases de refuser la professionnalisation :

«Moi, je ne veux pas avoir de patron. Au lieu de sortir gagnantes, nous allons y perdre. Si nous prenons 100, combien va-t-on vraiment toucher, $5 \%$ ? $10 \%$ ? Nous ne pourrons plus décider, il va décider notre paye. Aujourd'hui, si nous voulons, nous baissons le prix, ou nous l'augmentons. "Si tu travailles, tu dois faire tout ce que je te dis", dira le propriétaire, "tu dois être présente à telle heure". "Je vais vous payer telle somme chaque mois. Toi, tu vas coucher avec celui-ci ou celui-là”. Et toi, tu n'as plus le droit de réclamer. Pourquoi ? Parce que tu as signé un document. »

Comme celle de la COB qui a influencé ces discours, la conception du travail ici mobilisée est étroitement liée à l'imaginaire du salariat. Le réglementarisme en maison close limite en effet la possibilité d'envisager la prostitution comme une profession libérale. La position antiprofessionnalisation semblait gagner du terrain au sein des prostituées mais le rapprochement de certaines dirigeantes du collectif Mujeres Creando allait rapidement montrer les limites de son audience. Si les femmes des maisons closes avaient accepté de renoncer à être juridiquement reconnues comme travailleuses pour préserver leur autonomie, la mise en accusation des clients et le déni par les féministes du collectif de toute possibilité de subversion leur sembla inacceptable. Beaucoup se désolidarisèrent de leurs dirigeantes. Le refus pragmatique de la contractualisation ne contredisait pas l'intime conviction d'être des travailleuses du sexe. En revanche, en réfutant toute possibilité d'agency aux femmes, le filtre exclusif de la domination masculine oblitérait toute marge de manœuvre, condition nécessaire à l'expérience du travail y compris lorsqu'il est traversé par la domination ${ }^{24}$. La participation de nouvelles élues aux rencontres du réseau des travailleurs du sexe d'Amérique latine, parrainé par 1'ONUSida ${ }^{25}$, finit par inverser la tendance. En 2009, lors de la préparation du $2^{\mathrm{e}}$ congrès

national, les dirigeantes présentes décidèrent que toutes leurs revendications se feraient désormais depuis leur position de travailleuses sexuelles.

24. Natacha Borgeaud-GARCIANDA, "Travail, domination et sujet. Une réfléxion à partir des maquilas du Nicaragua et de leurs ouvrières ", in Natacha BoRGEAUD-GARCianda, Bruno Lautier, Ricardo Peñafield et Ania Tizziani (éds.), Penser le politique en Amérique latine. La recréation des espaces et des formes du politique, Khartala, 2009, p. 275-290.

25. La RedTraSex (Red de mujeres trabajadoras sexuales de America Latina y el Caribe) est née à la fin des années 1990. Situé en Argentine, son secrétariat est organisé par AMMAR (voir note 13). 


\section{Un travail sous conditions}

Que les femmes des maisons closes s'identifient comme travailleuses ne signifie cependant pas que leur position coïncide terme à terme avec la manière dont les analystes de l'OIT ou de l'académie peuvent entendre les arrimages de la prostitution au travail. Leurs outils conceptuels sont différents. Ils sont empruntés aux imaginaires du travail et des activités féminines des classes populaires des villes boliviennes. Yves Clot rappelait que le contenu et le sens du travail ne sont pas unitaires mais construits par sédimentation ${ }^{26}$. Dans le cas des prostituées rencontrées, ils conjuguent la question des revenus avec la logique, plus ancienne et moins marchande, de l'effort physique.

Ainsi, lors des entretiens réalisés en marge du processus d'organisation des associations de prostituées, la confusion du travail et de l'effort est apparue comme l'un des principaux obstacles à la construction de la prostitution comme travail. Sa présence se doit à la prégnance du monde agricole qui, via la migration, est à l'origine de l'actuel prolétariat urbain, dont les parents des prostituées. Nous l'avons évoqué avec le discours des syndicalistes, elle surgit d'une conception passive de la sexualité féminine qui lui vaut la qualification de « travail facile » et l'exclut de la sphère du travail archétypal. Voici par exemple comment Yuli envisageait la relation de la prostitution avec ce qu'elle entend par travail :

«Mon père avait un atelier de mécanique et c'est ce que j'ai vu toute ma vie. Tout ce que j'ai vu est avec du travail, avec de l'effort. Ce que je fais serait un effort mental, mais ce n'est pas un effort physique. C'est ça que moi j'ai vu. »

Travail et effort fonctionnent donc ici comme synonymes. D'autres témoignages confirment cette centralité de l'effort qui déplace jusqu'à la légalité. Après avoir souligné l'absence d'effort qu'elle associe avec son activité, Claudia évoque, par comparaison, les pichicateros [les fabricants artisanaux de cocaïne] :

«Si ça c'est du travail. Il [le pichicatero] n'est pas fainéant, assis toute la journée. S'il le mettait [le mélange à base de feuilles de coca] dans la machine et que la machine travaillait, il resterait les bras croisés, ce ne serait pas du travail. Mais eux, ils foulent, ils sont en mouvement, ils font des efforts, ils se sacrifient, c'est un travail. »

Fondé sur l'archétype du labourage, apanage des hommes, l'effort participe de la construction du travail comme un champ masculin et de la disqualification corrélative des activités féminines (et de leurs revenus).

26. Yves Clot, «Présentation », in Yves Clot (dir.), Les histoires de la psychologie du travail, Toulouse, Octarès, 1999. 
Ce principe au service d'une idéologie, hégémonique dans les strates populaires boliviennes, qui vise à préserver - ne serait-ce que symboliquement - le monopole des maris comme pourvoyeurs de l'économie $\mathrm{du}$ foyer. De fait, le commerce de rue vers lequel se tournent bon nombre de femmes mariées est lui aussi exclu de la catégorie travail en raison d'une absence supposée d'effort physique similaire à celle attribuée à la sexualité féminine. La conception de ses bénéfices comme une reproduction de capital, et non une production de richesses, parachève la déqualification de sa nature laborieuse ${ }^{27}$. De fait, les hommes qui n'ont pas d'autre choix professionnel préfèrent la vente ambulante - et de produits «masculins» comme les outils - pour compenser l'absence d'effort physique par la mobilité, autre qualité virile. La disqualification $\mathrm{du}$ travail et des revenus féminins est un symptôme classique de la domination masculine ${ }^{28}$. Le rôle central qu'y jouent l'effort ainsi que la référence au travail de la terre et à la production de biens matériels, fait écho aux théories des économistes physiocrates de l'Europe du XVIII ${ }^{\mathrm{e}} 29$ qui participèrent non seulement à l'infériorisation des femmes, mais aussi à celles des prêteurs et des artisans juifs dont les spécialités (tailleur, cordonnier...), qualifiées de faciles, étaient par là-même féminisées ${ }^{30}$.

L'exclusion de la prostitution du monde de l'effort laborieux est, j'allais écrire évidemment, l'explication vernaculaire classique de la surreprésentation des femmes des basses terres dans les maisons closes. En accord avec l'imagerie populaire de la nonchalance tropicale, Yuli présente ces dernières comme par nature peu enclines au travail et à l'effort :

«[Elles] sont fainéantes, elles n'aiment pas travailler, elles n'aiment pas laver, repasser..., c'est pourquoi, elles préfèrent l'argent facile de la prostitution. »

Ce faisant, notre interlocutrice reprend le reproche adressé par la population bolivienne à l'ensemble des femmes prostituées, quelle que soit leur origine. Ainsi, à la différence de ceux des commerçantes, les

27. Pascale ABSI, «Il ne faut pas mélanger les fortunes: Travail, genre et revenus chez les commerçantes de Potosi », in Valeria Hernandez, Pépita Ould-Ahmed, Jean PaPAIL et Pascale PhÉLInAs (éds.), Turbulences monétaires : L'Amérique latine dans une perspective comparée, L'Harmattan, 2007.

28. Jacqueline LAUfER, Catherine MARRY et Margaret MARUANI, Le Travail du genre. Les sciences sociales du travail à l'épreuve des différences de sexe, Paris, La Découverte, 2003.

29. Annie FouqueT, «L'invention de l'inactivité », Travail, genre et société, 11, 2004, p. 47-62.

30. Annie Benveniste, Le Bosphore à la Roquette. La communauté judéo-espagnole à Paris (1914-1940), L'Harmattan, 1989, p. 106-108. 
revenus des prostituées ne révèlent pas seulement des femmes qui ne se contentent pas du salaire d'un mari et entrent en concurrence avec lui. Ils témoignent d'une inadéquation plus générale aux rôles de genre qui autorise les femmes à n'aimer ni laver, ni repasser, dit Yuli... Ou, pour reprendre l'analyse de Paola Tabet, à dissocier relations aux hommes, obligations domestiques et sexualité au point de transformer cette dernière en service payant ${ }^{31}$. Parce que la déqualification du travail féminin passe in fine par celle de ses revenus (non laborieux, non productifs, etc.), c'est logiquement principalement sur ce terrain que se jouent les élaborations conceptuelles qui autorisent les prostituées à penser leur activité comme un travail.

Nous avons évoqué l'importance des revenus dans la qualification par les femmes des maisons closes de la prostitution comme travail. Le sens qu'elles donnent à l'équation des revenus et du travail se distingue cependant de la vision économiciste de l'OIT. Tandis que pour cette institution internationale tous les revenus se valent - dès lors qu'ils n'enfreignent pas les lois des pays qui la chapeautent - pour témoigner de l'existence du travail, le revenu qui définit ici la prostitution comme travail n'est pas une rémunération quelconque, neutre. Pour les prostituées, il ne suffit pas qu'il existe - encore moins, nous l'avons vu, qu'il soit légal - pour qu'un revenu acquiert valeur de travail. Il faut avant tout qu'il soit socialement affilié. Les femmes insistent toujours sur l'objectif familial de leurs revenus. Celles qui n'ont pas d'enfants, de mère ou de frères à entretenir sont mal vues dans le milieu et assignées à la catégorie « vicieuses » : une femme qui aime le sexe et les hommes. La distinction entre les revenus "professionnels» des femmes qui se sacrifient pour les autres (parents, enfants) et les revenus «non professionnalisant » des vicieuses contraste également avec les arguments féministes pour qui le revenu n'est pas un critère pour qualifier la prostitution. Que la plupart des prostituées s'auto-affilient à la première catégorie - celle des non-vicieuses - n'invalide pas la distinction. Ce n'est pas le revenu proprement dit qui convertit la prostitution en un travail (et la prostituée en travailleuse), mais la qualité de celle qui le gagne et la façon dont il est dépensé. Cette position n'est pas sans rappeler la démarche qui oppose aux théories orthodoxes du marché le fait que la valeur de l'argent est aussi construite par les rapports sociaux $^{32}$. Les prostituées s'accordent d'ailleurs sur ce point avec les

31. Paola TABET, La grande arnaque. Sexualité des femmes et échange économicosexuel, op. cit.

32. Viviana Zelizer, The Social Meaning of Money: Pin Money, Paychecks, Poor Relief and Other Currencies, Princeton University Press, 1994. 
tenants du camp anti-professionnalisation : pour qu'il y ait travail, il faut des rapports sociaux légitimes. Mais depuis quel point de vue ? Devenus expression d'altruisme, les revenus restituent aux femmes et à leur activité leur conformité aux normes de genre. Bien sûr, leur argent ne provient pas d'un seul mais de plusieurs hommes, mais leur destin n'est pas différent de celui d'une femme au foyer.

L'idée que les prostituées, qui sauvent des couples et freinent les viols, sont socialement utiles et pas seulement pour leur famille, qu'elles ne font rien de mal et gagnent honnêtement leur vie, permet d'opérer le glissement d'une activité légitimée par la maternité vers le monde du travail. La distinction avec le vol - tout au moins celui qui prend place hors des maisons closes - marque souvent cette frontière entre l'honnête et le malhonnête, le digne et l'indigne, le travail et le nontravail :

Daniela : «Pour moi, c'est un travail digne. Selon moi au moins. Parce que nous ne faisons pas pleurer [synonyme populaire de voler] les gens, nous n'allons pas voler dans les rues, nous sommes dans un lieu où on peut nous trouver [même lorsque nous volons], nous sommes comme cela.

- «Et voler, cest un travail?

«Pour moi, ce n'en est pas un, parce qu'ils [les voleurs] gagnent de ce qui est facile, ils font pleurer les gens, ce que nous ne faisons pas, n'est-ce pas ? Et tout ce qu'ils gagnent, ils le gaspillent...»

Non seulement les voleurs font «pleurer les gens » mais les bénéfices de leurs larcins qu'ils gaspillent, sous-entendu en boisson ou en filles, ne servent pas - à la différence des revenus des prostituées - à la reproduction socialement normée de la société.

Contrairement à ce que qu'on aurait pu attendre dans une société catholique comme la bolivienne, lorsque les prostituées évoquent le contenu moral de leur activité, elles ne font presque jamais allusion à la morale sexuelle (monogamie, norme affective et non négociable des rapports sexuels, procréation). Si l'argent de la prostitution est communément qualifié de "mal acquis » (plata mal habida), ou plus directement de «mauvaise vie» (mala vida) ou d'argent du diable, ce n'est pas en raison du commerce de services sexuels. Si l'argent de la prostitution est «mal acquis » c'est parce que « les hommes y dépensent l'argent de leur foyer, leurs pots-de-vin, ou ce sont des délinquants qui volent » explique Yuli, résumant l'avis général. De cette manière, le caractère malin de l'argent de la prostitution est antérieur à la transaction avec la prostituée. Il est dû à sa déviation, en amont, de la circulation monétaire légitime : les revenus masculins qui n'arrivent pas aux mains des épouses ou qui sont acquis par la corruption et le vol. Le destin familial que les prostituées revendiquent pour leurs revenus va alors 
moraliser l'argent des clients en le réinjectant dans les circuits de la circulation monétaire légitime. Jusqu'aux vols, qui font partie de la routine des services offerts aux clients et participent à ce processus de rédemption comme l'explique Poison :

«Ces hommes aussi, ils font pleurer leur femme. Pourquoi ne lui donnent-ils pas l'argent pour leurs enfants? Ils préfèrent venir ici pour faire les "grands" [seigneurs]... Et puis si ce n'est pas moi qui vole cet homme, ce sont les voleurs, les braqueurs qui vont le voler dehors et boire cet argent. Moi au moins, c'est pour donner à manger à mes enfants. »

Ainsi, c'est aussi parce qu'elle rétablit le destin légitime des revenus masculins et neutralise les écarts des hommes à leur propre rôle de genre, que la prostitution participe à la conservation de l'ordre social.

Tout en confirmant l'adéquation des prostituées avec les représentations de l'altruisme féminin, la conception de la prostitution comme sacrifice va permettre de contrecarrer le caractère facile de la prostitution et de ses revenus. L'évocation des longues veilles et le manque de sommeil, la consommation obligatoire d'alcool, la violence, le vieillissement prématuré et la dégradation du corps ainsi que la marginalité sociale prouvent que personne ne peut se prostituer par goût. Le sacrifice vient alors occuper le rôle structurel de l'effort dans la socialisation des revenus de la prostitution et donc sa constitution comme travail :

Norma : «C'est un travail parce que la raison qui nous fait entrer dans ce milieu, c'est la nécessité. C'est un travail fait de sacrifices. Il faut boire en accompagnant tous types d'hommes... C'est encore plus de sacrifices qu'une employée domestique, elles ont des pauses, nous nous risquons notre vie. »

Travail par ses revenus mais à condition qu'ils soient socialement affiliés et donc conformes aux rôles féminins ; non-travail par l'absence d'effort mais travail quand même lorsque le sacrifice remplace ce dernier: ces efforts d'élaboration intellectuelle qui visent à réduire les contradictions de l'affirmation de leur statut de travailleuse, n'a évidemment de sens que parce que les prostituées doivent sans cesse se justifier aux autres et à elles-mêmes. À l'image du « sale boulot ${ }^{33}$ » de leurs consœurs françaises, plus que les conditions concrètes d'exercice, le sentiment d'être jugées - mal - et leur marginalisation sont les principaux reproches que les prostituées boliviennes adressent à leur activité ; la principale raison pour laquelle elles souhaitent la quitter à moyen terme et en tenir éloignés leurs enfants. L'indignité de la

33. Stéphanie PRYEN, Stigmate et métier. Une approche sociologique de la prostitution de rue, op. cit. 
prostitution naît d'abord de ce regard extérieur explique Claudia en soulignant la relativité de ce concept :

« J'ai plusieurs amies qui travaillent comme balayeuses. À 5 ou à 6 heures du matin, elles sont en train de balayer, elles ramassent des ordures... [Ton un peu méprisant] Pour elles, c'est plus digne de ramasser des ordures, de ramasser des saloperies, parce qu'elles peuvent rentrer chez elles, être avec leurs enfants et leur dire : "Regarde, j'ai nettoyé, je suis fatiguée, aide-moi, d'accord ?" Mais moi, je ne parle à personne de mon travail, ni avec mes propres sœurs qui savent que je travaille. Je ne peux pas leur dire : "Regarde, tu sais, un client est venu et il m'a battue ou un client est venu et le préservatif s'est rompu". Je ne peux pas. [...] Les seules personnes avec qui je parle sont mes compagnes de travail...»

Ainsi, si ce n'était l'opprobre qu'elle suscite, aux yeux de Claudia la prostitution peut être même plus digne qu'un emploi de balayeuse. De fait, lorsqu'une mairie proposa à des prostituées d'intégrer des programmes municipaux de réfection des rues, beaucoup répondirent qu'elles «n'allaient pas se rabaisser à cela». Dans un monde où le travail constitue toujours le principal facteur de reconnaissance citoyenne et d'acquisition de droits sociaux ${ }^{34}$, la revendication des prostituées boliviennes prises dans les préjugés de leur société reste plus une pétition de reconnaissance qu'une proclamation idéologique. En Bolivie, rares sont les femmes qui présentent leur entrée en prostitution comme un véritable choix. Aucune ne revendique avec fierté une identité de travailleuse sexuelle.

\section{Pour conclure}

La place de la parole des prostituées et la possibilité qu'elle déjoue les manipulations dont elle est l'objet est l'enjeu central du débat international sur la professionnalisation. Le détour analytique par les représentations du travail montre cependant qu'y compris lorsqu'il y a accord sur la reconnaissance de la prostitution comme travail - et sur la possibilité de droits liés à ce statut - , des différences de fond subsistent sur la manière dont se construit cette identification.

Celles-ci ne tarderont pas à faire surface si le processus de professionnalisation poursuit son institutionnalisation portée par l'OIT, comme c'est le cas dans un nombre croissant de pays.

L'entrée par les représentations du travail est également complémentaire de celle sur les rapports de sexes, et la sexualité en générale, pour comprendre le statut social de la prostitution et donc la reconnaissance de ceux qui l'exercent. Dans un contexte où les femmes représentent tou-

34. Revue du MAUSS, «Travailler est-il (bien) naturel ? Le travail après la "fin du travail" ", 2001-2002, $\mathrm{n}^{\circ} 18$ 
jours la grande majorité des prostitués, la professionnalisation interroge aussi le travail des femmes. Dans cet article, j'ai esquissé la manière dont les prostituées boliviennes conceptualisent leur activité en regard de ce que leur milieu d'origine considère être le travail et notamment celui des femmes. Des mises en perspective similaires permettent au reste de la société de construire sa propre vision de la prostitution. On pressent ainsi que les crises qui affectent le monde du travail en général et le délitement des frontières de l'emploi salarié conventionnel visibilisent sans aucun doute les paramètres économiques de la prostitution ${ }^{35}$.

Enfin, le constat de la multiplicité des représentations du travail en jeu dans le débat sur la professionnalisation invite les analystes à s'arrêter sur leurs propres implicites qui, au-delà des postures personnelles, renvoient également à des traditions disciplinaires. Il n'est pas anodin de constater qu'alors que les sociologues s'interdisaient d'utiliser les outils de la sociologie du travail pour comprendre la prostitution - et donc d'écouter les voix de certains de ses praticiens les ethnologues construisaient des anthropologies du travail dans des sociétés où ce concept est inexistant. Qu'en est-il alors de l'objet travail qui définit le champ de la sociologie du même nom ? Malgré les efforts en direction d'une plus grande réflexivité, et la critique du concept informé par l'hégémonie du salariat, sa construction n'échappe pas aux visions du monde où elle se constitue et qu'elle vient légitimer en retour.

Institut de recherche pour le développement (IRD)

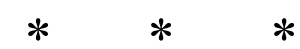

35. Ce qui invite, comme le propose Lilian Mathieu («La prostitution: zone de vulnérabilité sociale ", Nouvelles Questions Féministes, vol. 21, n² 2, 2002, p. 55-75), à comparer la prostitution dans ce qu'elle partage de structurel et d'inacceptable avec certains emplois précaires. 